\title{
Percepções da Morte entre os Estudantes Femininos recém ingressos do Curso de Medicina, Durante Pandemia de Covid 19
}

\section{Perceptions of Death among Newly Entered Female Medical Students During the Covid 19 Pandemic}

DOI: $10.46919 / \operatorname{archv2n1-010}$

Recebimento dos originais: 30/10/2020

Aceitação para publicação: 23/12/2020

\author{
Valentina Vetorasso Cabrera Mano \\ Graduando em medicina - 3 período \\ Instituição: Centro Universitário Barão de Mauá - Ribeirão Preto \\ Endereço: Rua Florêncio Rosário 225 \\ E-mail: valentinavetorassocabrera@gmail.com \\ Júlia Lascala Cardoso \\ Graduando em medicina - 3 período \\ Instituição: Centro Universitário Barão de Mauá - Ribeirão Preto \\ Endereço: Rua Floriano Peixoto, 1591 Brodoski \\ E-mail: jujulascala@hotmail.com \\ Maria Fernanda Maluf Novaes Franco \\ Graduando em medicina - 3 período \\ Instituição: Centro Universitário Barão de Mauá - Ribeirão Preto \\ Endereço: Rua Florêncio Rosário 175 \\ E-mail: mariafernanda.vest19@outlook.com
}

\author{
Adriana Novaes Rodrigues \\ Doutora em Ciências Da Saúde \\ Instituição: Universidade de São Paulo - SP
}

Endereço: Avenida Doutor Arnaldo, 455 - $1^{\circ}$ andar - Cerqueira César - São Paulo - SP -Brasil

\section{RESUMO}

O presente artigo aborda, através da análise de gênero e de uma reflexão ética, o medo da morte durante a pandemia de Covid 19, nos recém chegados aos cursos de medicina. atribuição do ato de cuidar às mulheres na sociabilidade burguesa. Objetivo: problematizar as percepções da morte pelo estudante recém egressos a faculdade de medicina e recursos no enfrentamento da pandemia da COVID-19. Método: Questionário firmado especificamente para estudo e uma reflexão teórica baseada na literatura científica da área. Resultados: Entre as principais aplicações dessa dimensão no contexto de atenção em saúde destaca-se como recurso de enfrentamento e fonte de apoio o assunto morte e como suporte nas situações de isolamento social e quarentena. Conclusão: Recomenda-se que novos estudos e novas explicação das repercussões emocionais da doença, diante de um cenário pandêmico impermanente.

Palavras-chave: Pandemias, Religião, Espiritualidade, Infecções por Coronavirus.

\section{ABSTRACT}

This article addresses, through gender analysis and an ethical reflection, the fear of death during the Covid pandemic 19, in newcomers to medical courses. attribution of the act of caring for women in bourgeois 
sociability. Objective: to problematize the perceptions of death by the student who recently graduated from medical school and resources in facing the pandemic of COVID-19. Method: Questionnaire signed specifically for study and a theoretical reflection based on the scientific literature of the area. Results: Among the main applications of this dimension in the context of health care, the subject of death stands out as a coping resource and source of support and as support in situations of social isolation and quarantine. Conclusion: It is recommended that further studies and new explanations of the emotional repercussions of the disease, in the face of an impermanent pandemic scenario.

Key words: infections, Pandemics, Mental health

\section{INTRODUÇÃO}

A doença, popularmente conhecida como coronavírus (COVID-19), é caracterizada pela síndrome respiratória aguda grave causada pelo coronavírus 2 (SARS-CoV-2), que se transformou em pandemia 2019. Em relação a população, os profissionais de saúde são um grupo impactados emocionalmente pelos aspectos da COVID-19, pois estão diretamente ligados ao combate desta patologia. Xiang, et al (2019), evidenciaram que os profissionais de saúde, são vulneráveis a alto risco de infecção e problemas de saúde mental, além do medo da morte em suas famílias, amigos ou colegas 1

A morte é um evento biológico natural e inevitável da vida humana, que gera medo na maioria das pessoas, por significar o desconhecido. Muitas culturas ao longo da história, buscam através dos mitos, das religiões e das representações nas artes e na filosofia, a fim de minimizar a angústia gerada pela morte 2 . Porém, a representação da morte é individual, construída através de diferentes formas, por meio da cultura, das tradições familiares, ou, mesmo, pela investigação pessoal 3.

A pandemia e as modificações das rotinas diárias e as medidas adotadas para contê-la parecem impactar na saúde mental, aumentando o risco para surgimento de sintomas de estresse, ansiedade e depressão, em todos os seguimentos da população 4. Neste contexto, a COVID-19 pode ser considerada não só uma patologia epidemiológica, mas, também psicológica 5, devido as alterações cognitivas, emocionais e comportamentais desencadeadas nesse período6.

O recém chegado a escola de medicina, deparou com uma inusitada situação, com aulas não presenciais, trazendo suas angústias e medos causadas pelo afastamento social e a crise de mortes causada pelo Covid 19.

A falta de experiencia na prática clínica, o medo de contágio, a possibilidade de perdas familiares são itens que diretamente influem e fluem na rotina do estudante de medicina, que ingressou durante a pandemia.

Diante do exposto, o objetivo do presente estudo é sistematizar conhecimentos sobre os processos de medo de contágio e morte no contexto da pandemia de COVID-19, buscando caracterizar demandas psicológicas nos alunos emergentes das faculdades de medicina e discutir implicações na prática diária. 


\section{MÉTODO}

Trata-se de um estudo observacional e transversal. Utilizou-se um questionário estruturado, contendo 10 questões, abertas sobre dados epidemiológicos e visão da morte e perdas familiares durante a pandemia, vivenciados e sumarizados através das experiências dos autores, considerando as restrições impostas frente à COVID-19. A abordagem qualitativa, e de caráter descritivo, é um método de investigação que enfatiza a compreensão da experiência humana como é vivida, através da coleta e análise dos materiais narrativos, subjetivos, onde se permite a compreensão dos comportamentos das pessoas em determinadas situações 7 .

Para melhor sistematização do conhecimento, baseado em Ferrari, (2015), foi realizado uma revisão de literatura, visto que tal estratégia se mostra adequada para tópicos ainda pouco explorados, oferecendo subsídios para o desenvolvimento de novas modalidades de intervenção 8. Os buscadores utilizados nas bases de dados indexadas, Medical Publications [PubMed], ScienceDirect, Google Scholar e Scientific Electronic Library Online [SciELO]), foram “end-of-life”, “death", “coronavirus" e "COVID-19”.

Os dados foram armazenados em banco de dados no programa Excel.

\section{RESULTADOS}

Em relação aos dados epidemiológicos, a idade média dos participantes foi 19,6 anos, pertencentes ao terceiro período do curso de medicina, recém ingressados em uma faculdade particular da cidade de Ribeirão Preto. Em relação ao sexo todos os participantes foram do sexo feminino.

Em relação aos padrões medo, afastamento social e busca de apoio durante a pandemia, estão expressos no gráfico 1 .

Quando ao item de possuir em casa pessoas do grupo de risco, tecnicamente $100 \%$ dos entrevistados disseram que possuíam.

Em relação a religiosidade como pilar de sustentação psicológica, durante a pandemia, 100\% das participantes apresentaram alguma expressão religiosa, independente do rótulo religioso atribuído. 
Gráfico 1 - Visão sobre a morte

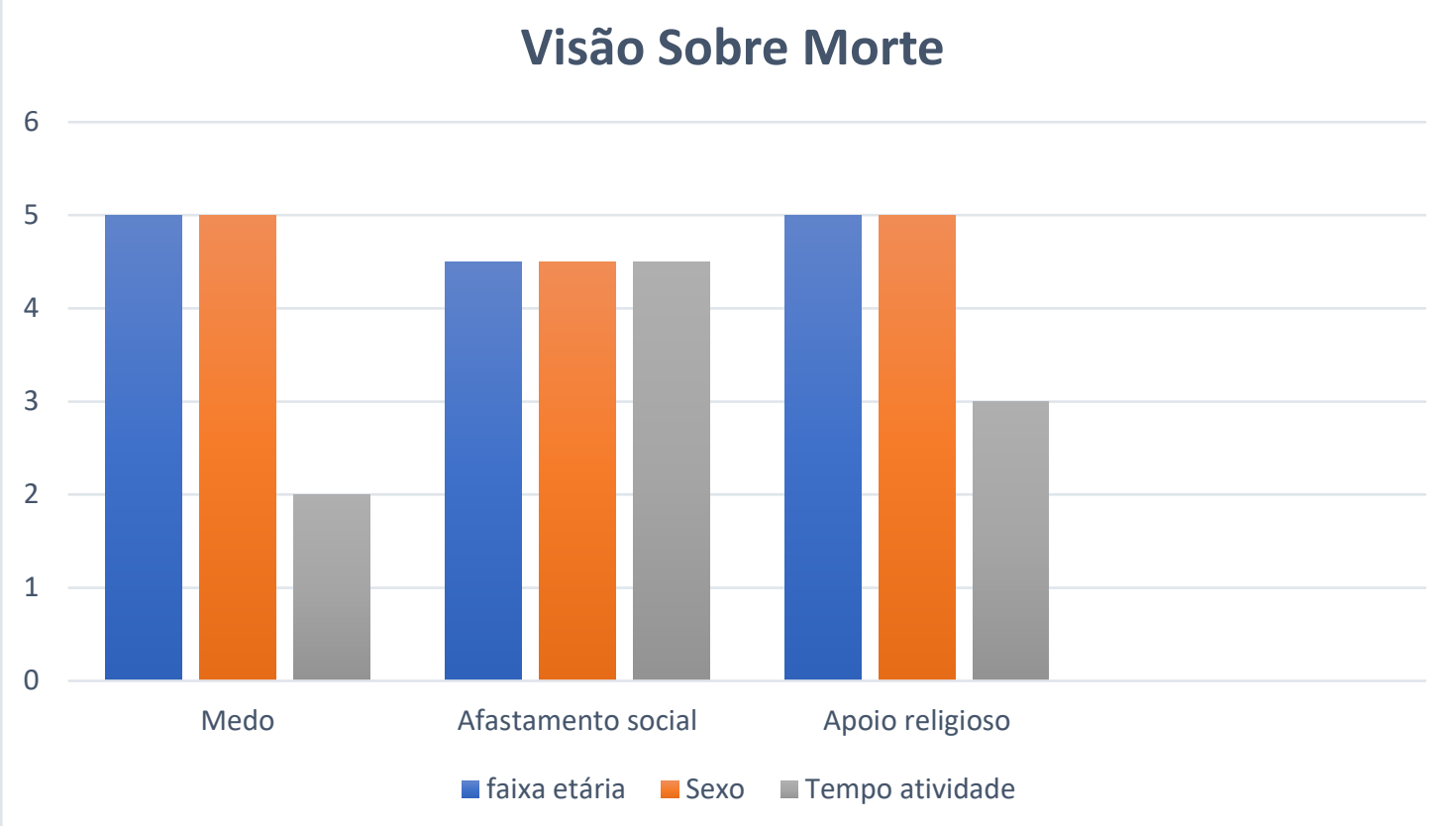

A morte em relação ao medo, afastamento social e apoio durante pandemia Covid 19

\section{COMENTÁRIOS}

O médico tem a sua formação voltada para o tratamento e o diagnóstico das doenças, no entanto, ele tem de rever os seus conceitos e conhecer seus limites durante a graduação, pois as demandas de conhecimentos e experiencias envolvem também os fatores psicológicos, social e espiritual.

Dos alunos que tiveram contato com a morte na graduação, a maioria afirmou ter mudado sua visão durante a atuação, como acadêmicos, e muitos deles afirmaram querer conhecer mais o tema e compreender melhor a terminalidade da vida 10

A ideia de que a religião tem como objetivo aplacar os temores diante do ininteligível 9e a religião teria finalidades utilitárias, tendo a função de organizar defesas contra os medos do aniquilamento 11.

O mais intrigante de todas as questões abordadas com os graduandos foi um tema polêmico, mas necessário durante a vivência sobre a morte durante a pandemia - o que há após a morte? Podemos observar que o apoio espiritual foi essencial aos entrevistados. Ao tentar explicar como se dá "o futuro após a morte", a busca e apoio através da religião foi trazido em todas as respostas, inclusive para suas angústias e medos, o mesmo apoio que Kübler-Ross (2008) relata em suas pesquisas 12.

O contato com a morte pelo estudante de Medicina acontece ao longo do curso, mas os graduandos recém ingressos no curso, durante a pandemia por Covid 19, encontraram um panorama muito diferente dos anos anteriores. Eles não tiveram o primeiro contato com experiencias dos veteranos, nem tiveram a percepção direta da morte. O contato com o tema foi introduzido através da própria pandemia, que como 
todas as pandemias anteriores, acarretou um grande número de mortes em um curto espaço de tempo, o que traz implicações psicológicas diversas 13.

No caso da COVID-19, em particular, algumas medidas adotadas para conter a rápida escalada do número de infectados, através do distanciamento social, 14 dificultam interações face a face entre os alunos e a formação de uma rede socioafetiva, através membros acadêmicos da faculdade 15.

A escolha de participantes do sexo feminino foi embasada historicamente referida a mulher, de que ela tem uma sobrecarga subjetiva, mas realista, quando se refere à percepção e reação emocional dos familiares 16.

A revisão da literatura realizada, segundo Pautasso, (2020), permite a integração de estudos sobre um mesmo assunto, o que favorece as reflexões sobre a problemática, cujas pesquisas são recentes e adotam diferentes delineamentos, como é o caso da pandemia de COVID-19 17.

Apesar do afastamento social, as informações vindas através dos meios de comunicação, do grande número de vidas perdidas e dos grupos de riscos, além das alterações das rotinas, têm sido vivenciadas pelas mudanças rápidas das rotinas diárias, gerando insegurança e medo com o futuro imprevisível 18, o que foi detectado no grupo investigado. Esta insegurança pode ser explicada por dois fatores que priorizam o lado psicológico do estudante, sendo eles o medo e a possível perda de entes queridos. Estes dois fatores são relacionados ao fato de possuírem pessoas de grupos de risco em seu meio familiar, o que é confirmado pelos relatos de Arango, (2020). 19

Diante dessa realidade os estudantes novatos da área da saúde tornam-se mais vulneráveis aos sentimentos que se relacionam a finitude da vida.

Assim, estudos que se inserem nessa temática se justificam, pois, apesar de potencialmente enriquecedoras, as relações interpessoais foram afetadas pelo afastamento social e o medo da possibilidade da perda por morte de familiares. Podemos observar que a religiosidade expressa papel importante, como pilar de "sobrevivência psicológica" dos participantes deste estudo.

\section{CONCLUSÕES}

Os objetivos deste trabalho foram alcançados, visto que ficou evidente que a morte tem uma percepção, dos futuros médicos, muito superficial e possibilitou obter maior conhecimento acerca das percepções sobre a morte entre os estudantes femininos, recém ingressos ao curso de medicina, durante Pandemia de Covid 19.

O medo da morte, influenciou as condutas sanitárias através do afastamento social, decorrente dos grupos de risco para covid 19, em meio familiar. 
Estudos futuros devem aprofundar este conhecimento, através de pesquisas longitudinais que possibilitem investigar variações sobre o medo da morte, em ambos os sexos, no decorrer do curso de medicina e suas consequências psicológicas.

Há uma necessidade ainda presente de estudar mais e investigar a influência da religião, sobretudo nas experiências humanas relacionadas a morte durante a pandemia. 


\section{REFERENCIAS}

Xiang Y-T, Yang Y, Li W, Zhang L, Zhang Q, Cheung T, et al. Timely mental health care for the 2019 novel coronavirus outbreak is urgently needed. Lancet 2020;7(3):228-9. DOI: 10.1016/S22150366(20)30046-8

Rockembach JV, Casarin ST, Siqueira HCH. Morte pediátrica no cotidiano de trabalho do enfermeiro: sentimentos e estratégias de enfrentamento. Rev. Rene. Fortaleza. 2010; 11(2):63-71.

Eizirik CL, Polanczyk GV, Eizirik M. O médico, o estudante de medicina e a morte. Rev AMRIGS. 2000; 44(1-2):50-5.

Wang, S. S., Teo, W. Z., Yee, C. W., \& Chai, Y. W. (2020). Pursuing a good death in the time of COVID19 [Ahead of Print]. Journal of Palliative Medicine. https://dx.doi.org/10.1089/jpm.2020.0198).

Weir, K. (2020a, April, 1). Grief and COVID-19: mourning our bygone ives. American Psychological Association. Retrieved from https://www.apa.org/news/apa/2020/04/grief-covid-19.

Enumo, S. R. F., Weide, J. N., Vicentini, E. C. C., Araujo, M. F., \& Machado, W. L. (2020). Enfrentando o estresse em tempos de pandemia: proposição de uma cartilha. Estudos de Psicologia (Campinas), 37, e200065. http://dx.doi.org/10.1590/1982-0

Oliveira SL. Tratado de metodologia científica: projetos de pesquisa, TGI, TCC, monografias, dissertações e teses. São Paulo: Pioneira Thomson Learning; 2002. 320p.

Ferrari, R. (2015). Writing narrative style literature reviews. Medical Writing, 24(4), 230-235. https://dx.doi.org/10.1179/2047480615Z.000000000329

Kovács MJ. Morte e existência humana: caminhos de cuidados e possibilidades de intervenção. Rio de Janeiro: Guanabara Koogan; 2008

Chagas RRS, Castro CF, Moura VKST, Cavalcante JC, Cavalcanti SL, Freitas DA Percepções da Morte entre os Estudantes de Medicina Revist. Port.: Saúde e Sociedade. 2016;1(3): 217-227

Scorsolini-Comin F, Rossato L, Cunha VF, et al. A Religiosidade/Espiritualidade como Recurso no Enfrentamento da Covid-19. Revista de Enfermagem do Centro Oeste Mineiro. 2020;10:e3723. DOI: http://doi.org/10.19175/recom.v10i0.3723

Kübler-Ross E. Sobre a morte e o morrer. Trad. Paulo Menezes. 9a ed. São Paulo: Martins Fontes; 2008. Taylor, S. (2019). The psychology of pandemics: preparing for the next global outbreak of infectious disease. Newcastle upon Tyne: Cambridge Scholars Publishing

Ferguson, N., Laydon, D., Nedjati Gilani, G., Imai, N., Ainslie, K., ... Ghani, A. (2020). Report 9: impact of Non-Pharmaceutical Interventions (NPIs) to reduce COVID19 mortality and healthcare demand. London: Imperial College. Retrieved from http://hdl.handle.net/10044/1/77482

Ho, C. S., Chee, C. Y., \& Ho, R. C. (2020). Mental health strategies to combat the psychological impact of COVID-19 beyond paranoia and panic.Annals, Academy of Medicine, Singapore , 49(1), 1-3. Retrieved from http://www.anmm.org.mx/descargas/Ann-Acad-Med-Singapore.pdf 
Tessler RC, Gamache GM. Family experiences with mental illness. Westport: Auburn House; 2000.

Pattison, N. (2020). End-of-life decisions and care in the midst of a global coronavirus (COVID-19) pandemic.Intensive and Critical Care Nursing, 102862, 1-3. https://dx.doi.org/10.1016/j.iccn.2020.102862

Weaver, M. S., \& Wiener, L. (2020). Applying palliative care principles to communicate with children about COVID-19 [Ahead of Print]. Journal of Pain and Symptom Management. https://dx.doi.org/10.1016/j.jpainsymman.2020.03.020

Arango, C. (2020). Lessons learned from the coronavirus health crisis in Madrid, Spain: How COVID-19 has changed our lives in the last two weeks [Ahead of Print].Biological Psychiatry. https://dx.doi.org/10.1016/j.biopsych.2020.04.003 